\title{
From microfluidics to nanofluidics and signal wave- guiding for nanophotonics, biophotonics resolution and drug delivery applications
}

\author{
Salinas $\mathbf{C}^{1}$ and Bracamonte $\mathrm{AG}^{1,2 *}$ \\ ${ }^{1}$ Instituto de Investigaciones en Físicoquímica de Córdoba (INFIQC), Departamento de Química Orgánica, Facultad de Ciencias Químicas, Universidad Nacional \\ de Córdoba. Ciudad Universitaria, 5000 Córdoba, Argentina \\ ${ }^{2}$ Departement de chimie et Centre d'optique, photonique et laser (COPL), Université Laval, Québec (QC), G1V 0A6, Canada
}

\begin{abstract}
The aim of this Review was to show based on recent microfluidics developments how it could be incorporated within new Advanced Clinical Research for Future Nanomedicine treatments the design of versatile Micro and Nanodevices. These developments were done by a control of the Nanoscale for applications from Nanophotonics to Genomics, Biophotonics and Drug Delivery studies accompanied with enhanced Imaging resolutions and faster diagnosis and treatments. In addition, it was discussed the last Noble Prize in Physics 2018 related with Laser phenomena and development of instrumentation for Medical applications as individual Biostructure trapping and eye chirurgical interventions. By this manner the main goal of this short Review was to give an opinion about the state of the Art of Microfluidics to Nanofluidics with incorporation of Lasers in different optical approaches, applications and Future perspectives.
\end{abstract}

\section{Introduction}

In this Review it was discussed and showed how from accurate control of Nanoparticles synthesis, for Nanophotonics and Biophotonics, joined to in Flow methodologies it could be tracked from Micro-structures and Nanoplatforms for Biodetection, Biological event tracking and molecular detection.

In flow methodologies as Cytometry and Imaging Cytometry [1] were used for different applications as Cell counting, Biostructure discrimination, and it was even applied for immune-phenotyping to trigger signal processing [2]. This available Instrumentation in Research and Clinical Laboratories showed to be an important tool. However enhanced detection approaches are of high interest based on the combination of targeted Multifunctional Nanoparticles, and In Flow methodologies. But, new In Flow methodologies are in progress as Microfluidics and Nanofluidics coupled to Advanced Optical instrumentation as well. These reduced sizes channels of few micrometers to nanometres diameters and millimetres lengths, permitted the synthesis and incorporation of reduced sizes of Nanoparticles within confined volumes for enhanced resolutions. In this manner it was required lower samples volumes and smaller quantities of reagents for improved resolutions depending of needs and Optical set-ups applied.

Moreover, based on needs of different signal detection and transductions for all the mentioned applications it was discussed Enhanced Wave signal propagation within Waveguides for Micro- and Nanodevice fabrications.

In addition it was discussed Futures perspectives of these In Flow Advanced methodologies from Nano-Optical applications as characterizations of new Nanomaterials to Precision Medicine applications as Genomics, Neurophotonics, and controlled Drug Delivery within the Brain.

\section{Control of the nanoscale}

The control of the Nanoscale by different méthodologies, as for example in colloidal dispersion by chemical methodologies and over surfaces with high Energy Laser applications as Micro and Nanolithography techniques; permitted the fabrication of Multi-functional Nanoplatforms and Patterned surfaces.

It is of our interest the design and synthesis of Nanoparticles with different chemical modifications accompanied with enhanced Luminescent properties and targeted functionalities as Biomolecular detection, Biolabelling and Biostructure tracking within Nanophotonics and Biophotonics Fields [3]. In particular, it was of our interest the development of silver and gold Core-shell nanoparticles with Ultraluminescent properties applied for DNA detection and tracking of individual Biostructures based on Metal Enhanced Fluorescence (MEF). This phenomenon consisted in the generation of high Energy electromagnetic fields from the metallic surfaces by Laser excitation, named as Plasmons, which produced increased excitation of the fluorescent reporters at close distances within intervals of distances of nanometer $(\mathrm{nm})$. In order to control these lengths were applied Silica shells, organic polymer spacers, Biomolecules, and antibodies, etc.

*Correspondence to: A Guillermo Bracamonte, $\mathrm{PhD}$, INFIQC, Institute of Physicochemical Research of Cordoba, Department of Organic Chemistry, National University of Cordoba (UNC), Argentine, E-mail: gbracamonte@fcq. unc.edu.ar; guillermobrac@yahoo.ca

Key words: microfluidics, nanofluidics, waveguides, nanophotonics, biophotonics, genomics, biomolecular detection and drug delivery

Received: February 08, 2019; Accepted: February 25, 2019; Published: March 02, 2019 
The strategies assayed for molecular detection was the application of Fluorescent labellers, Plasmonic recognition and signal transduction within the near field and Fluorescence Resonance Energy Transfer (FRET) coupling as well. But in addition to these phenomena it was required different spectroscopic techniques, methodologies and specific optical instrumentation set ups for signals tracking. By this manner for faster diagnosis and treatments the generation of Bioimaging with enhanced In Flow resolution based on Fluorescence was used.

\section{In flow cytometry coupled to lasers and enhanced fluo- rescence and imaging detection}

In Flow methodologies as Cytometry showed to be very useful for cell, bacteria counting and Biological event tracking as well; but the incorporation of imaging detection systems showed better results. However, the incorporation of optimized optical lens within In Flow Cytometry systems coupled to cameras for Imaging recording permitted improved single nanoparticles tracking and small Nanoaggregates formed by few nanoparticles analysis. Based on this Enhanced resolution Imaging flow cytometry (IFC) experimental set-up [4], as for example for genomics applications, it was detected the SRY gen related with sex-genre determination [5] (Figure 1).

Moreover, it was of our interest the design and synthesis of Silica Nanoplatforms with supramolecular systems, used as mimetics systems of antibodies antigens interactions, by molecular complexation of targeted Organic Fluorescent molecules. Then, it was evaluated their applications for Fluorescent molecular reporter detection by commercial In Flow Cytometry with Laser excitation and Fluorescence detection on Individual Nanoplatforms. In this manner, it was observed a linear relationship between the signal recording and Fluorescent event counting from variable concentrations added of the targeted Fluorescent reporter within a colloidal dispersion of modified Silica Nanoplatforms. It was demonstrated how these Nanoarchitectures could be applied in Flow methodologies to Biomolecular tracking applications. Further experiments are in progress in order to validate this mentioned proof of concept for biomolecules and labelled Biostructures.

It should be highlighted that from the practical point of view In Flow methodologies showed to be low consumable cost, low real sample volumes required, with faster detection for early diagnosis. For these reasons from the use of Micro-capillaries by In Flow Cytometry [6] to the use of controlled reduced sizes of microfluidics to Nanofluidics dimensions new approaches of instrumentations coupled to different optical set-ups are in progress depending of requirements of samples and properties to be measured accompanied with higher Analytical performances.

\section{Microfluidics}

Different materials and Fabrication methodologies were developed for Microfluidics fabrication. It could be mentioned soft lithography and PDMS (polydimethylsiloxane) as most applied technique and material used respectively. However, it was applied many other organic polymers and hybrid layers deposition depending of needs and applications too. In this manner lower volume channels were designed and joined by different bonding techniques. Sizes from few to hundreds of micrometres and variable designs from crossing channels to multi-channels Chips, on silanized substrates of few $\mathrm{cm}^{2}$ surfaces, with variable volumes for different variable time of residence of the fluids were reported. And by this way as for example few microliters $(\mu \mathrm{L})$ as $2.0-5.0 \mu \mathrm{L}$ intervals, it was possible passed through

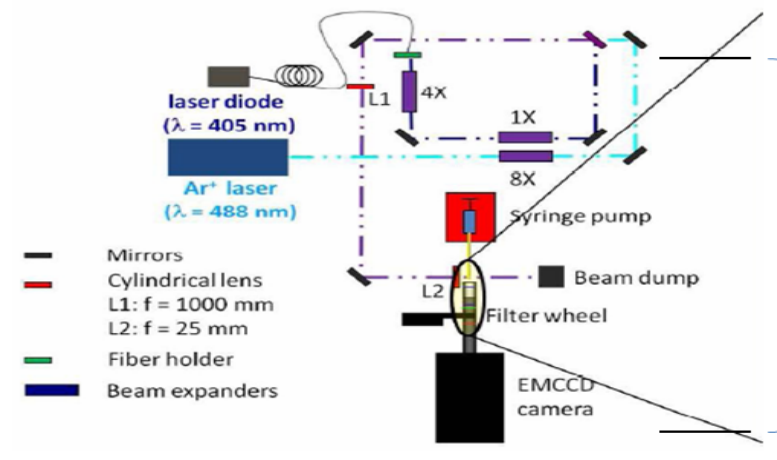

(i)

(i)
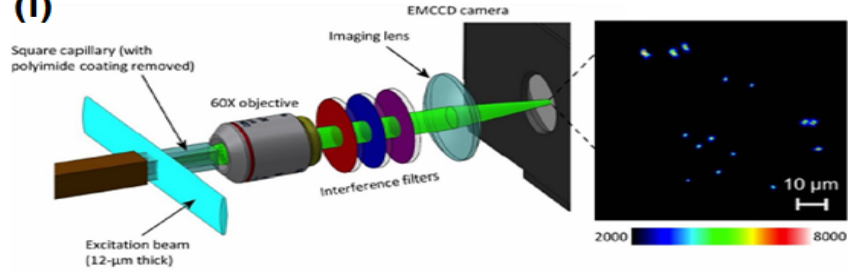

Figure 1. Scheme of Enhanced resolution Imaging flow cytometry (IFC) experimental setup. Inset Image corresponded to DNA detection by Metal Enhanced Fluorescence (MEF) from Core-shell and Core-less nanoparticles . Reprinted with permission from Ref 2 (Denis Boudreau, et al.). Copyright 2011 ACS Nano
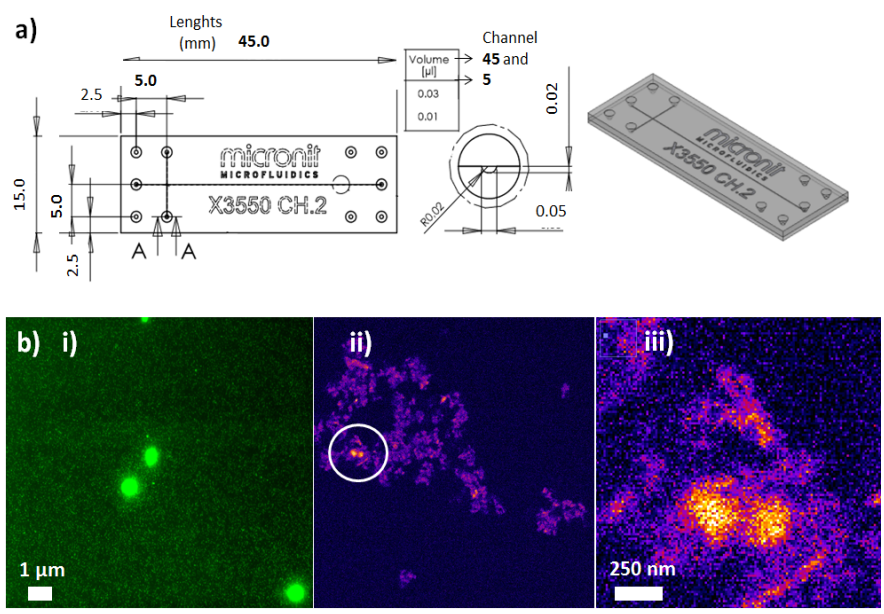

Figure 2. a) Scheme of Cross Channel Microfluidic Chip. b) Inset Images corresponded to (i) Ultraluminescent gold Core-shell Silica NPs and (ii) and (iii) modified Silica nanoparticles with supramolecular systems as mimetics of antibodies antigens interactions for targeted Organic Fluorescent molecular reporter complexations and detection by Laser Fluorescence Microscopy. Reprinted with permission from, Micronit Microfluidics Company and A. Guillermo Bracamonte, et al., respectively. Copyright 2019

these channels (Figure 2). In these dimensions, small Nanoparticles could be passed through for different types of studies (Figure 2) as well as Biostructures as Cells, Bacteria, Virus, etc.

Based on these Chips it was reported many applications; but it could be highlighted the following recent examples from the application of these microdevices with micro-pumps and optical detections systems coupled or incorporated in standard commercial instrumentation as In Flow Cytometry as well. It could be mentioned, from the synthesis of nanoparticles within confined spaces with minimal volumes of reactants [7], to bioanalytical flow cells for biofilm studies [8], Microfluidic flow confinement to avoid chemotaxis-based upstream growth [9], and Bioanalytical applications as Biostructure Counting [10], and Biological events detection as well [11]. However, 
the reduction of even smaller volumes was desired and required for highly accurate Optical Nanomaterial characterizations, and improved resolutions for tracking of individual events. By this manner higher accurate methodologies for fabrication of In Flow channels within the nanoscale were developed and they are currently used in highly accurate and confined studies. Moreover, new potential In Flow studies based on individual Biostructures tracking, analysis and counting based on Bacteria labelling with Ultraluminescent gold Core-shell Nanoparticles [12] and genetically modified Fluorescent Bacteria grafting with silver nanoparticles [13] by MEF Imaging detections would be of high impact as well (Figure 2).

\section{Nano-fluidics}

Fabrication of Nanofluidic devices involucrated dimensions below $100.0 \mathrm{~nm}$, and by this manner within lower volumes, it was obtained higher volume and surface ratios from individual Nanoarchitectures or small Nanoaggregates where the chemical interactions and new properties at short length scales had taken higher relevancies [14].

These confined spaces showed to be very useful for studies of new material properties and applications where a highly accurate control of volumes, concentrations and flows should be applied. However, within this field there are many Research projects and Funding in progress that it will provide knowledge accompanied with new insides and breakthroughs ideas for a large number of Life Science Research as well as from applied Nano- to Micro-technologies within different Research areas.

As for example it could be mentioned studies of Nano-Optics within low volumes for individual nanoparticles analysis and tracking. Recently it was reported a real time Plasmonic lasing from Nanocavities arrays incorporated within Nano-channels [15] (Figure 3). This study showed the potential of analysis within the near field for MEF [16] applications as tool of Bio-assays based on single Nanoplatforms analysis where it could be targeted few molecules at Single Molecule Detection (SMD) [17-20] level. As for example, for new developments of DNA detection for Genomics based on SMD approaches by Single Nanospectroscopy it could be applied. By this manner Potential
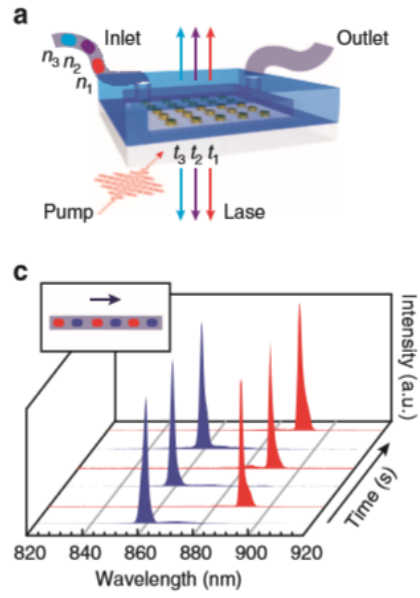

b

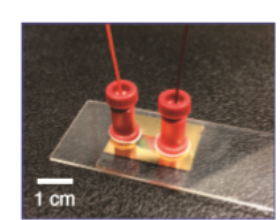

d

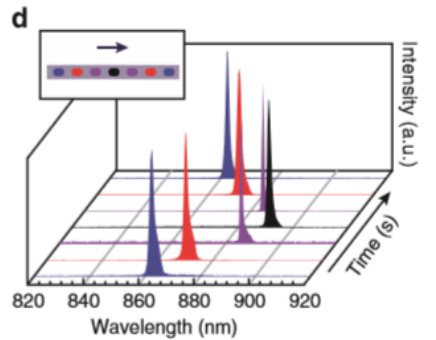

Figure 3. Lasing emissions from Au Nanoparticles arrays tuned in real time. a) Scheme of the dynamic laser; b) photograph of the device; c) switching lasing wavelengths and; d) shifting lasing wavelengths. Note: In c, IR-140 dye molecules were dissolved in DMSO (blue) and BA (red). In d, IR-140 dye molecules were dissolved in DMSO (blue), DMSO:BA=2:1 (red), DMSO: BA=1:2 (purple) and BA (black). DMSO:dimethyl sulfoxide, BA: benzyl alcohol. The emission intensities were normalized. Reprinted with permission from Ref 13 (Teri W. Odom, et al.). Copyright 2015 Nature applications from In Flow methodologies already studied and validated as free PCR detection of SRY gen in real blood samples [21] based on Enhanced Luminescent Nanoplatforms by MEF tracking would be of high impact as well (Figure 3).

Moreover, within confined spaces in the Nano-scale were Infrared Spectroscopically tracked water Molecular interactions based on a Plasmonics-Nanofluidics hybrid device [22]. This configuration permitted to confine and let the molecules interact with a high Energy Plasmonic array that it generated molecular-Plasmonic coupling from the modified vibronic modes. In this manner it was elucidated the $\mathrm{H}$-bonds network in comparison to bulk water. This communication showed the high sensitivity of the Plasmonic interactions joined to In Flow Nanofluidics from just water hidrogen bonding. Potential future applications for Biomolecular interactions are of high interest.

In addition, these confined volumes could be used as highly accurate controlled targeted drug and Nanoparticles delivery. As for example, from neurological disorders and needs of novel therapeutic solutions, it was opened the interest of low volume administrations of drugs by an accurate and precise administration. By this way, controlled Drug delivery within mouse brain by low volume injections based on Microfluidics channels close to the Nanoscale was recently reported [23]. This high impact approach was based on a neural probe incorporating a microfluidic ion pump for on-demand drug delivery and electrodes for recording local neural activity. This delivery approach enabled precise drug release into the brain region of a rodent model of epilepsy with negligible local pressure increase. This In Flow approach could be applied as well for new Nanomedical treatments with controlled targeted Cargo Nanoparticles administrations and incorporation of Optrodes for neuronal signaling tracking [24]. In addition, another example based on the versatility of Chip fabrication and their incorporation within standard instrumentation, as for example it should be highligthed the nano flow cytometer for single lipid vesicle analysis [25]; that it could be expanded to another Nano and Microstructures of Biological interest. At this point it should be mentioned as example the importance of targeted Exosomal RNA particles detection, involucrated in long-distance intercellular communications in diabetes [26] and cancer [27].

So, in this manner it was showed the huge potential and large number of applications from the Micro to the Nano-InFlow methodologies in progress. Future advanced applications of high impact it should be developped in the next years.

\section{Plasmonic and enhanced fluorescence wave-guiding}

The generation and detection of different wave signals, from communication to sensing applications based on Waveguides, is still being a challenge due to signal losses within the conductive materials. This observation generated special interest of enhanced signal studies from new Hybrids materials, Meta-materials and instrumental set-ups approaches.

In this field Silicon Photonics showed to be largely applied for different Micro-devices fabrications. These devices were based on their intrinsic properties of Silicon related with Optical transparent properties accompanied with semi-conductive characteristics in controlled conditions. As for example it could be mentioned Silica Waveguides, as modified silica surfaces to conduct signals within patterned guides. In this type of devices, the signal transduction occurred through planar modified surfaces; and by this manner the transduction of detected light signals of targeted wavelengths generated from the Nano-scale 
could be transmitted through mili-meters lengths. However, in order to avoid signal losses, it should be specially considered the modification of the Silicon channels with different conductive materials depending of the targeted signals as well as of the design of the guide channels.

About fabrication and modification of Silica waveguides, it could be mentioned new Metamaterials by Resonant Plasmonic approaches with Core-shell nanoparticles for Fluorescence signals routing with potential applications for Biomolecular sensing within microdevices [28]. In addition, these Metamaterials applied for Silica Wave-guides have high impact within studies of Quantum optical circuits as well as for Routing, and Detection of Resonance Fluorescence recently reported on On-Chips [29] based on semi-conductive materials.

Moreover, it was reported recently strongly Confined Surface Plasmon Polaritons Waveguiding Enabled by Planar Staggered Plasmonic Waveguides [30]. In this manner, it was arrived to signals transductions and detections in the Far Field from generated Electromagnetic fields within the near Field of metallic surfaces within Silica Wave-guides by Laser excitation. The mentioned near and Far field were related with shorter distances at the Nanoscale and longer-ones respectively. These Advanced signals Wave-guiding Research approaches are in progress and potential high impact applications for new analytical and Bioanalytical methodologies are desired and required. However, it should be highlighted that the signal propagation and transduction occurred in one dimension within patterned waveguides were applied through microfluidics channels in contact with conductive modified surfaces for fabrication of standard Spectroscopical instrumentation that today is commercially available. This Spectroscopic Technique is known as Optical Waveguide Lightmode Spectroscopy (OWLS). This technique could be used from In Flow surface characterization studies [31] to Cell and Molecular detection [32] and adsorption of proteins detection as well [33] (Figure 4).

Just to finish it should be mentioned that many techniques for fabrication of Silica Wave-guides are based on different Lithography techniques with multi-layered depositions over silica substrates and surface modifications by Laser etching and electro-beam irradiations [34]. The control in the Fabrication of these devices arrived to the design of Silicon Photonic Wire Waveguides [35], where a Silica core was surrounded by silica-based cladding within dimension below 500 $\mathrm{nm}$ of diameters that enhanced the refractive index, improved the total internal reflection and diminished the signal loss with a similar approach to Optical fibers that it could be applied to accurate and targeted sensing applications.

So, based on the different mentioned Research directions new approaches of signal transductions based on Silicon Meta-materials are

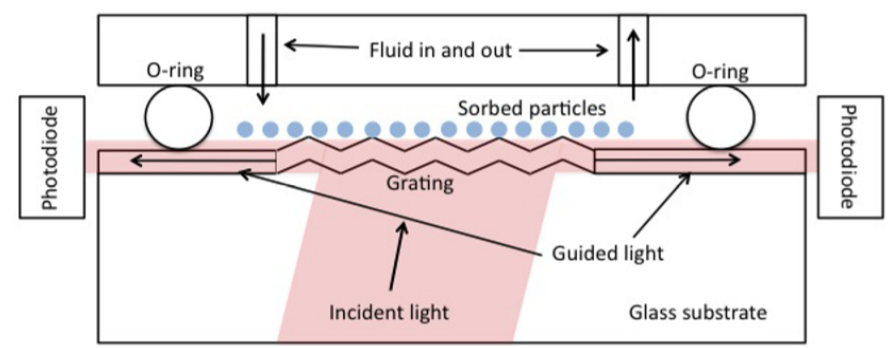

Figure 4. The physical configuration of OWLS. The angle at which incident light is guided with constructive interference and signal transduction to the photodiode detectors is used to calculate the effective refractive index of the waveguide. Reprinted with permission from Ref 25 (Jinke Tang, et al.). Copyright 2012 Sensors attended to be developed in the next years for high impact applications in different Research Fields.

\section{Laser and instrumentations from the Nobel Prize of Physics 2018}

The aim of this section was to discuss and highlight the importance of the applications of Lasers, not only for Microfluidics to Nanofluidics fabrications, but as well for source of high Energy photons for Biophotonics and Clinical Research and New Medical treatments. And within this Research Field, especially this year it should be mentioned the Nobel Prize of Physics 2018 for the developments of Tools made of light [36] shared by USA, France and Canada, as Optical tweezers $[37,38]$ and generation of high-intensity, ultra-short optical pulses $[39,11]$, ; with many applications.

These applications were based on Laser Instrumentation with high Energy photon generation comparable to $\mathrm{x}$ - and gamma rays energies that demonstrated dense matter and even nuclear structures characterization, to be applied for Cancer treatments, Optical Biostructure trapping, Bioimaging, Ultrafast Images of matter, Eye surgeries and commercial electronic devices.

This invention was named as Chirped Pulses Amplified (CPA) characterized by the generation of short pulses below 1 ps with energies at the Joule level by stretching of a short, low energy pulse within a single optical fiber. This pulse was linearly chirped in the fiber by combination of group velocity dispersion and self-phase modulation. The stretched pulse was obtained by amplification and compression by a double grating compressor [40]. In this manner it could be obtained fully compressed pulses that allowed higher energies to be achieved before self-focusing occurs (Figure 5). In this scheme the chirp was illustrated with colours. Initially the gain medium (amplifier) was Nd:glass, whereas today, another common gain medium is Ti:sapphire. The impact of shortened pulses below ps permitted higher improved resolution by impact of higher Energy photons. Within the applications it could be mentioned the related with Life-Sciences as for example the manipulation of Single Living Cells Using Infra-Red Laser Beams [41] and eye surgeries [42]. The application of these Ultra-fast short pulsed Lasers permitted to improve and avoid collateral damages from their intrinsic higher Energy focused beam that permitted enhanced resolution and accuracy on their dissections in "Cataratas" surgeries (Figure 5).

\section{Future perspectives of inflow micro and nanofluidics within nanophotonics, biophotonics and nanomedicine}

From the Known-how related with the accurate control of the Microscale to the Nanoscale with High Energy Lasers over chemically modified surfaces by different methodologies it was possible the design of variable Micro- and Nano-patterning. Based on these designs, depending of the materials used, it was arrived to different applications. One of them, it was the fabrication of controlled sized confined channels for sensing and drug delivery. Then it was coupled to different Optical approaches for excitation of the sample to collect information from their intrinsic material properties of the Fluids and new detection approaches as well. These synthesized Micro and Nanodevices, today are largely applied for Research and Developments applications accompanied with incorporation in already developed In Flow methodologies where the known-how of fabrication of capillary channels coupled to Optical systems as well took the main 


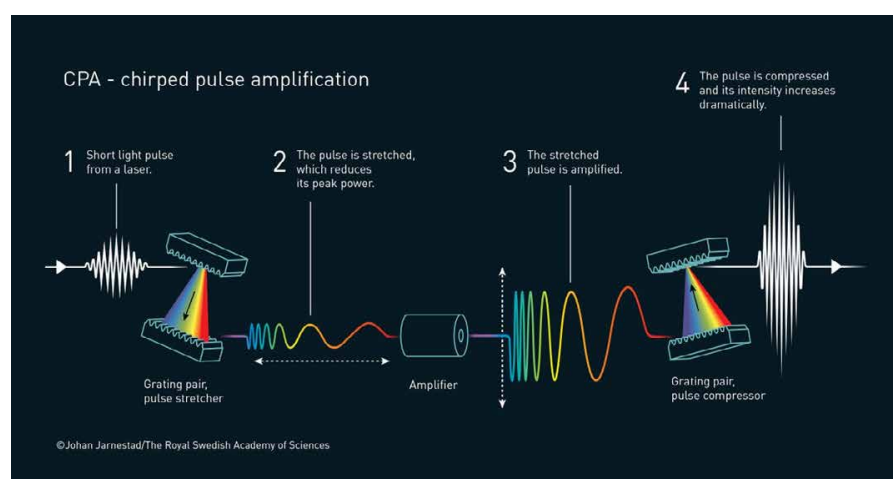

Figure 5. The CPA technique revolutionised laser technology. It enabled the emission of very intense, short pulses of light using an intricate method to avoid the risk of destroying the amplifying material. Instead of amplifying the light pulse directly, it is first stretched in time, reducing its peak power. Then the pulse is amplified and when it is compressed more light is collected in the same place - the light pulse becomes extremely intense. Reprinted with permission from Ref 30 (Arthur Ashkin, Gérard Mourou and Donna Strickland et al.). Copyright 2018 Royal Swedish Academy of Sciences

relevance. And it was already demonstrated their incorporation on real applications with the consequent impact on the market as for example by In Flow Cytometry instrumentation [43].

So, From the Research and Development point of view, the question that it should be answered is what it will be developed next. But, as it was discussed in this Review application related with Nanostructures synthesis, Nano-optics studies based on In Flow single nanoparticles analysis are of high impact. Moreover, these low volume Flows permit to be proposed for advanced Research applications as controlled drug delivery. This application challenge, even the brain barriers that opened legal debates from mouse to primates applications due to the accurate tissue cut where each mm counts for penetration of the Micro-, Nanodevice in the cranial space. In addition, to these devices could be coupled to Opto-sensors for Real time Sensing applications.

Moreover, the Biomolecular In Flow sensing applications on Lab.On particles to study molecular and molecular-cell interactions, at low concentrations and Single Molecular (SMD) Detection levels, have a high impact on Future applications. In addition enhanced signal transduction should be developed within surface Waveguiding for Micro-devices fabrications, that they could be easily coupled to all In Flow methodologies.

These In Flow confined volume approaches are possible with low volume uses, fast detection and tracking, and low costs due to their required small reagent quantities. In addition the relative easy accessibility to design your own In-Flow Chips by the use of Kits for fabrications provided by commercial Companies increase the accessibility and possibilities of new studies. In addition their incorporation in standard instrumentation of Research and Clinical Laboratories, coupled with advanced Optical approaches, it will permit new approaches for advanced studies that it will open new debates and discussion about their real applications as well. As for example, from Genomics applications it could be mentioned available DNA detection boxes for forensic applications offered for the most known and important companies that opened law debates as well. In this manner, we are just in the beginning of a huge Research field with high Science, Industry and Social impact.

\section{Acknowledgment}

We would like to gratefully acknowledge the different Grants received in order to accomplish the Research work in progress of the authors in this field, CONICET, Consejo Nacional de Investigaciones Científicas y Técnicas (National Research Council of Argentine); ANPCyT, Agencia Nacional de Promoción Científica y Tecnológica (National Agency of Scientific and Technology Promotion of Argentine); and especially to SECyT (Secretary of Science and Technology from the National University of Cordoba (UNC), Argentina, for awarding us the extension of the Grant for Young Researchers at INFIQC.

Especially thanks to Professor Denis Boudreau from Département de chimie and Centre d'optique, photonique et laser (COPL), Québec, Canada, for the long standing Research Collaboration in progress; as well as to all the Canadian Grants that permit it. Finally specially thanks to Lucia V. Bracamonte and Rosalia F. Bracamonte for the English revision.

\section{References}

1. Barteneva NS, Fasler-Kan E, Vorobjev IA (2012) Imaging flow cytometry coping with heterogeneity in biological systems. J Histochem Cytochem 60: 723-733. [Crossref]

2. Lin P, Owens R, Tricot G, Wilson CS (2004) Flow cytometric immunophenotypic analysis of 306 cases of multiple myeloma. Am J Clin Pathol 121: 482-488. [Crossref]

3. Salinas C, Bracamonte G (2018) Design of advanced smart ultraluminescent multifunctional nanoplatforms for biophotonics and nanomedicine applications. Frontiers in Drug, Chemistry and Clinical Research 1: 1-8.

4. Brouard D, Viger ML, Bracamonte AG, Boudreau D (2011) Label-free biosensing based on multilayer fluorescent nanocomposites and a cationic polymeric transducer. ACS Nano 5: 1888-1896. [Crossref]

5. Brouard D, Ratelle O, Bracamonte AG (2013) Direct molecular detection of SRY gene from unamplified genomic DNA by metal-enhanced fluorescence and FRET. Analytical Methods 5: 6896-6899.

6. Austin Suthanthiraraj PP, Graves SW (2013) Fluidics. Curr Protoc Cytom Chapter 1: Unit 1. [Crossref]

7. Leester-Schadel M, Lorenz T, Jurgens F, Richter C (2016) Fabrication of microfluidics devices, microsystems for pharmatechnology, springer international publishing switzerland A. Dietzel (ed.), Chapter 2.

8. Li W, Greener J, Voicu D, Kumacheva E (2009) Multiple modular microfluidic ( $\left.\mathrm{M}^{3}\right)$ reactors for the synthesis of polymer particles. Lab Chip 9: 2715-2721.

9. Pousti M, Zarabadi M, Amirdehi MA, Paquet -Mercier F, Greener J (2019) Microfluidic bioanalytical flow cells for biofilm studies: A review. Analyst 144: 68-86.

10. Asayesh F, Zarabadi MP, Aznaveh NB, Greener J (2018) Microfluidic flow confinement to avoid chemotaxis-based upstream growth in a biofilm flow cell reactor. Anal. Methods 10: 4579-4587.

11. Yi Yang S, Hsiung SK, Hung YC (2006) A cell counting/sorting system incorporated with a microfabricated flow cytometer chip. Meas. Sci. Technol 17: 2001-2009.

12. Skommer J, Akagi J, Takeda K, Fujimura Y, Khoshmanesh K, et al. (2013) Multiparameter Lab-on-a-chip flow cytometry of the cell cycle. Biosens Bioelectron 42: 586-591. [Crossref]

13. Gontero D, Veglia AV, Boudreau D, Bracamonte AG (2018) Ultraluminescent gold core-shell nanoparticles applied to individual bacterial detection based on metalenhanced fluorescence nanoimaging. J. of Nanophotonics 12: 1-12.

14. Golberg K, Elbaz A, McNeil R, Kushmaro A (2014) Increased bioassay sensitivity of bioactive molecule discovery using metal-enhanced bioluminescence, Nanopart Res 16: $1-14$.

15. Duan C, Wang W, Xie Q (2013) Review article: Fabrication of nanofluidic devices, Biomicrofluidics 7: 1-41.

16. Yang A, Thang B Hoang (2015) Real time tunable lasing from plasmonic nanocavity arrays. Nature Communications 6: 1-7.

17. Gontero D, Veglia AV, Bracamonte AG, Boudreau D (2017) Boudreau, Synthesis of ultraluminiscent gold core-shell nanoparticles as nanoimaging platforms for biosensing applications based on metal enhanced fluorescence. RSC Adv 7: 10252-10258.

18. Punj D, de Torres J, Rigneault H, Wenger J (2013) Gold nanoparticles for enhanced single molecule fuorescence analysis at micromolar concentration, Optics express 21 : 27338- 27343 . 
19. Betzig E, Hell SW, Moerner WE (2014) The nobel prize in chemistry 2014, for the development of super-resolved fluorescence microscopy, Press release from the royal swedish academy of sciences.

20. Puchkova A, Vietz C, Pibiri E, Wünsch B, Sanz Paz M (2015) DNA origami nanoantennas with over 5000 -fold fluorescence enhancement and single-molecule detection at $25 \mu \mathrm{M}$. Nano Lett 15: 8354-8359.

21. Karim F, Smith TB, Zhao C (2017) Review of optical detection of single molecules beyond the diffraction and diffusion limit using plasmonic nanostructures. $J$. Nanophotonics 12: 1-15.

22. Brouard D, Ratelle O, Perreault J, Boudreau D (2015) PCR-free blood group genotyping using a nanobiosensor, VoxSanguinis. International Journal of Transfusion Medicine 108: 197-204.

23. Le HH, Morita A, Mawatari K, Kitamori T, Tanaka T (2018) Metamaterials-enhanced infrared spectroscopic study of nanoconfined molecules by plasmonics-nanofluidics hydrid device. ACS Photonics 5: 3179-3188.

24. Proctor CM, Slézia A, Kaszas A, Ghestem A (2018) Electrophoretic drug delivery for seizure control. Sci. Adv 4: 1-8.

25. Dufour S, De Koninck Y (2015) Optrodes for combined optogenetics and electrophysiology in live animals. Neurophotonics 2: 1-14.

26. Friedrich R, Block S, Alizadehheidari M, Heider S (2017) A nano flow cytometer for single lipid vesicle analysis. Lab Chip 17: $830-841$

27. Santulli G (2018) Exosomal microRNA: The revolutionary endogenous oofstdf nanotechnology. Sci. Trans. Medicine 10: 1-4.

28. Sun Y, You S, Tu H, Spillman DR, Channey EJ, et al. (2018) Intraoperative visualization of the tumor microenvironment and quantification of extracellular vesicles by label free nonlinear imaging, $S c i A d v$ 4: 1-10.

29. Grégoire A, Boudreau D (2017) Chapter 28: Metal-Enhanced fluorescence in plasmonic waveguides, springer science business media dordrecht B. Di Bartolo et al. (eds.), Nano-Optics: Principles Enabling Basic Research and Applications, NATO Science for Peace and Security Series B: Physics and Biophysics. DOI 10.1007/97894-024-0850-8 28.

30. Reithmaier G, Kaniber M, Flassig F, Lichtmannecker S, Müller K, et al. (2015) On-chip generation, routing, and detection of resonance fluorescence. Nano Lett 15: 5208-5213. [Crossref]
31. Ye L, Xiao Y, Liu Y, Zhang L, Cai G (2016) Strongly confined spoof surface plasmon polaritons waveguiding enabled by planar staggered plasmonic waveguides. Scientific Reports 6: 1-8.

32. Yu H, Eggleston CM, Chen J, Wang W, Dai Q, et al. (2012) Optical waveguide lightmode spectroscopy (owls) as a sensor for thin film and quantum dot corrosion. Sensors 12: 17330-17342.

33. Gunawan RC, King JA, Lee BP (2007) Surface presentation of bioactive ligands in a nonadhesive background using dopa-tethered biotinylated poly(ethylene glycol). Langmuir 23: 10635-10643.

34. Vörös J (2004) The density and refractive index of adsorbing protein layers. Biophys $J$ 87: 553-561. [Crossref]

35. Righini GC, Chiappini A (2014) Glass optical waveguides: a review of fabrication techniques. Optical Engineering 53: 1-14.

36. Lockwood DJ, Pavesi L (2011) Silicon photonics II. Topics in applied physics 119, K. Yamada, Chapter 2: Silicon photonic wire waveguides: Fundamentals and Applications $1-29$.

37. Ashkin A, Mourou G, Strickland D (2018) The noble prize in physics 2018, for tools made of light: ground-breaking inventions in the field of laser physics. Press Release from The Royal Swedish Academy of Science.

38. Ashkin A (1970) Acceleration and trapping of particles by radiation pressure. Physical Review Letters 24: 156-157.

39. Ashkin A, Dziedzic JM (1975) Optical levitation of liquid drops by radiation pressure. Science 187: 1073-1075. [Crossref]

40. Strickland D, Mourou G (1985) Compression of amplified chirped optical pulses. Optics Communications 56: 219-221.

41. Maine P, Strickland D, Bado P, Pessot M, Mourou G (1988) Generation of ultrahigh peak power pulses by chirped pulse amplification. IEEE Journal of Quantum Electronics 24: 398-403.

42. Ashkin A, Dziedzic JM (1989) Optical trapping and manipulation of single living cells using infra-red laser beams. Ber. Bunsenges. Phys. Chem 93: 254-260.

43. Palanker D (2018) Femtosecond lasers for ophthalmic surgery enabled by chirpedpulse amplification. The New England Journal of Medicine 379: 2267-2269.

Copyright: (C2019 Salinas C. This is an open-access article distributed under the terms of the Creative Commons Attribution License, which permits unrestricted use, distribution, and reproduction in any medium, provided the original author and source are credited. 